\title{
Agôn
}

Revue des arts de la scène

$8 \mid 2019$

Matières

\section{Peinture et écritures scéniques contemporaines : la peinture-matière chez Jan Fabre, Romeo Castellucci et Vincent Macaigne}

\section{Kenza Jernite}

\author{
(2) OpenEdition \\ Journals \\ Édition électronique \\ URL : http://journals.openedition.org/agon/6332 \\ DOI : $10.4000 /$ agon.6332 \\ ISSN : 1961-8581 \\ Éditeur \\ Association Agôn
}

Référence électronique

Kenza Jernite, «Peinture et écritures scéniques contemporaines : la peinture-matière chez Jan Fabre, Romeo Castellucci et Vincent Macaigne ", Agôn [En ligne], 8 | 2019, mis en ligne le 09 décembre 2019, consulté le 03 juin 2020. URL : http://journals.openedition.org/agon/6332 ; DOI : https://doi.org/ 10.4000 /agon.6332

Ce document a été généré automatiquement le 3 juin 2020.

Association Agôn et les auteurs des articles 


\title{
Peinture et écritures scéniques contemporaines : la peinture- matière chez Jan Fabre, Romeo Castellucci et Vincent Macaigne
}

\author{
Kenza Jernite
}

1 À la fin des années 1990, Roger Planchon prédit l'avènement du règne de la peinture au théâtre en ces termes :

Je vois la peinture qui essaie de sortir du cadre et je sens que l'écriture scénique, qui va surgir sous des formes diverses, sera une aventure de la peinture, la peinture sortie du tableau. Une révolution picturale, comme la découverte de la peinture abstraite par rapport à la peinture figurative. On s'éloigne brusquement du texte. Le spectateur passe du temps mental à l'espace mental. Une Révolution' ${ }^{1}$.

2 La peinture - comme modèle heuristique et esthétique - va en effet peu à peu envahir la scène, des tableaux wilsoniens aux investigations plastiques de Romeo Castellucci. En particulier, au tournant des années 2000, la peinture sort effectivement du cadre pour se déverser en liberté sur les scènes de certains metteurs en scène européens. Entre 2002 et 2004, Romeo Castellucci crée, dans différentes villes d'Europe, sa Tragedia Endogonidia, où la peinture rouge coule à flots, alors que le metteur en scène explore les liens qui peuvent se tisser entre sang de la tragédie grecque et martyr christique. En 2006, Vincent Macaigne présente un Requiem tout aussi chargé en fausse hémoglobine. Dans la mise en scène qui suit ce premier spectacle, Idiot! en 2009, le déversement de peinture est désormais intégré à la grammaire macaignienne ; ce n'est plus seulement le faux sang qui est déversé, mais tout devient pigment (on pense en particulier à la scène lors de laquelle Aglaïa renverse sur le prince Mychkine un seau de peinture verte), et tout est menacé de retourner au chaos, dans ce versement incontrôlé. Le faux sang coule aussi en liberté dans la cour d'honneur du Palais des Papes, lorsque Jan Fabre y présente son Je suis Sang, en 2001. Il sera remplacé par des trombes d'eau en 2005 dans son Histoire des Larmes, puis finalement par des flots de peinture dans Mount Olympus, en 2017. J'appelle peinture-matière cette peinture liquide et encore informe 
qui se met alors à se déverser sur les scènes contemporaines, matière faite de pigments colorés pouvant être mélangés, comme nous avons commencé de le voir, à différents liquides.

Cette arrivée de la peinture-matière sur les scènes contemporaines dans les années 2000 correspond à un regain d'intérêt, dans le champ des arts plastiques, pour les explorations de certains artistes de performance des années 1970. Les Actionnistes viennois - chez qui la peinture, mélangée aux fluides corporels, coule à flots - sont en particulier mis à l'honneur. Une première exposition au Musée du Louvre en 2000, "Posséder et Détruire "2, leur fait une grande place; elle est suivie fin 2001 d'une exposition intitulée «La peinture comme crime " ${ }^{3}$, toujours au Musée de Louvre, où l'on retrouve, en plus des archives des performances des Actionnistes viennois, des sections consacrées aux œuvres de Klein, ou encore de Jackson Pollock. Il semble fondamental de reconnaitre ce dialogue qui se tisse entre les artistes de performance des années 1970 et les metteurs en scène du début du XXI ${ }^{e}$ siècle si l'on veut comprendre les divers enjeux que recouvre l'arrivée - voire chez certains l'envahissement - de cette peinturematière sur la scène. Ce qui se jouait chez les artistes de performance est en effet repris et réintégré dans les écritures scéniques qui nous occupent.

On remarque en particulier chez les artistes de performance venus des arts plastiques cette tendance à faire couler la peinture, ou à la remettre en liberté. Chez des artistes européens comme Gina Pane ou encore Herman Nitsch, cette peinture est peu à peu assimilée aux humeurs corporelles - elle les figure ou elle s'y mêle - elles-mêmes souvent ramenées, dans des pratiques qui tiennent du rituel, aux sanies christiques. Les artistes américains qui jouent avec cet écoulement de peinture ont plutôt tendance quant à eux à suivre les questionnements esthétiques et plastiques de leurs prédécesseurs, en particulier ceux des expressionnistes abstraits qui libèrent la main en même temps que la peinture ; il s'agit pour une artiste comme Carolee Schneemann de désosser la toile pour travailler séparément ses différents composants, pour Jim Dine d'interroger son obsession pour le pigment.

5 Cependant, d'un continent à l'autre, le dénominateur commun de ces actions est bien une volonté de s'affranchir des règles, qu'elles soient esthétiques, morales, ou sociales. L'écoulement de peinture, dans ce qu'il a d'immaitrisable, devient le geste par excellence de ces tentatives d'affranchissement. Comme l'écrit l'historien de l'art Guillaume Cassegrain dans son ouvrage sur la coulure :

L'écoulement, les écoulements sous toutes leurs formes possibles (organiques, chimiques) sont devenus avec certaines manifestations modernes d'engagements artistiques - les happenings, notamment - un argument expressif qui, sans être systématiquement isolé et magnifié à l'intérieur d'un discours global, parvenait à exprimer efficacement les notions de dépense, d'excès si importantes pour ces actions aux visées subversives. «Je répands, j'asperge, je souille l'espace avec du sang ", déclaration d'intention d'Hermann Nitsch pour l'une de ses "actions", s'appuie sur cette force symbolique de la matière libérée de toutes contraintes d'usage. Le liquide dispersé sans retenue dans l'espace où se déroule l'événement, les traces laissées par la violence des jets de matière sur les murs, le sol ou les corps redonnent à la substance (peinture, sang, urine, selles) une fonction transgressive. La liquidité de la peinture que l'art classique a niée par tout un ensemble de pratiques (séchage, glacis) et de notions esthétiques (temps de réflexion et de finition qui assurent au peintre la réalisation d'une œuvre «finie») est soudainement rappelée par l'écoulement d'une matière fluide qui ne peut plus être contenue et canalisée ${ }^{4}$. 

en scène qui choisissent d'entrer en dialogue avec la peinture et d'en faire spectacle : on rappelle que nombre d'entre eux, de Fabre à Castellucci, ont aussi une formation d'artistes plasticiens. Ils intègrent dans leurs écritures scéniques l'écoulement de peinture, et donc les significations qui lui sont attachées par les artistes de performance que nous avons évoqués : sidération, souillure, révolte... Mais ils jouent aussi des capacités capillaires de cette peinture à l'état brut: chez Jan Fabre par exemple, dans un spectacle comme Je suis Sang, la peinture rouge, qui apparaît pour la première fois lors d'un combat chevaleresque, figure la blessure, puis, alors qu'elle envahit les culottes et les robes de mariées des performeuses, est associée au sang menstruel, avant d'envahir la totalité du plateau, dans une évocation sanguinaire de l'épisode du Déluge. On le voit, les significations de cette peinture-matière peuvent varier d'un spectacle à l'autre, mais également au sein même d'un spectacle.

Quoi qu'il en soit, à chaque fois que la peinture se trouve convoquée sous sa forme la plus brute, elle vient questionner, voire mettre en danger le cadre - qu'il s'agisse du cadre du tableau, du cadre scénique ou des cadres de pensée et d'écriture. Cette intrusion de la peinture-matière comme tache, rature, voire souillure, n'est cependant bien souvent qu'une première étape. Comme dans le Je Suis Sang de Jan Fabre, la peinture, dans les spectacles qui nous occupent, finit bien souvent par envahir le plateau, en une reprise du thème diluvien, qui, selon les spectacles, renvoie tour à tour à l'Apocalypse ou à la Genèse. Comme de nombreux peintres avant eux (de Turner à Cézanne), les metteurs en scène ici étudiés, en utilisant la peinture comme liquide et matière, ne cessent d'en revenir à la question du commencement du monde et de sa représentation.

\section{Révolte, blasphème, résistance : la peinture comme élément perturbateur}

8 La peinture, sous sa forme liquide et brute, arrive presque toujours sur scène comme élément perturbateur. Le déversement de matière sur scène doit être mis en lien avec une forme d'irrévérence, de révolte voire de blasphème.

\section{Les révolutions ratées de Vincent Macaigne}

Dans la dramaturgie macaignienne, où les coulées de peinture se mêlent aux déversements d'eau ou de terre, la peinture est ainsi synonyme de révolte.

À l'automne 2017, Vincent Macaigne présente au théâtre des Amandiers Je suis un pays, fable apocalyptique qui suit le destin des enfants de la Terre, Marie et Heidi. Alors qu'Heidi-CEdipe s'empare du pouvoir, devient tyran et prend part à toutes les compromissions possibles, Marie (violée par un Ange au début du spectacle dans une parodie de l'Annonciation, et qui porte la parole nouvelle) fait figure de révolutionnaire et tente par tous les moyens de faire tomber son frère. Ce spectacle doit être mis en regard d'un second spectacle de Vincent Macaigne présenté au même moment à la Villette, et intitulé En Manque. La fable oppose cette fois, toujours dans une atmosphère de fin du monde, une richissime collectionneuse qui habite sur l'olympe avec les plus riches, et qui a rassemblé tous les chefs-d'œuvre de la peinture européenne, et sa fille, 
représentante des pauvres (les habitants de la vallée), membre d'un groupe révolutionnaire, qui a juré de tuer sa mère et de détruire la fondation. Les deux spectacles sont très proches non seulement par les thèmes qu'ils mettent en œuvre - la révolte des faibles contre les puissants - mais aussi par la grammaire esthétique qu'ils déploient. Dans les deux spectacles, les plateaux accueillent des reproductions de tableaux du Caravage: dans En Manque, c'est le David avec la tête de Goliath (c. 1609), exposé sur le mur du fond, qui prend la place la plus grande, en une reprise explicite du thème du spectacle qui oppose les gens d'en bas aux puissants; dans Je Suis un Pays, ce sont des reproductions de L'Amour victorieux (c. 1601-1602) et du Crucifiement de saint Pierre (1600) qui font face aux spectateurs (mais d'autres tableaux du Caravage sont également présents sur les murs latéraux). Dans les deux spectacles, ces tableaux vont ensuite disparaître - la toile de fond sur laquelle étaient reproduits les tableaux dans Je suis un Pays monte dans les cintres, tandis que les reproductions de tableaux sont détruites sous les yeux des spectateurs dans En Manque - au profit d'une peinture brute qui envahit peu à peu la scène.

Dans Je suis un Pays, alors qu'il a été décidé que de nouvelles élections se tiendraient dans le royaume et qu'a lieu le premier débat présidentiel de ces élections, Marie, qui a rejoint un groupe rebelle, fait irruption sur scène et renverse un seau de peinture noire sur la présentatrice du débat télévisé - seau qui, si l'on se fie aux indications figurant dans le texte de Macaigne, est censé être rempli d'excréments ${ }^{5}$. Par ailleurs, une fois enfermée dans une cage avec le «roi immortel» (dont Heidi a pris la place), Marie tente de l'assassiner encore et encore à grands jets de peinture rouge, qui se trouve dans des bouteilles et dans des pots, placés avec Marie dans la cage. Encore une fois, il n'est pas fait mention de peinture dans le texte; nous pouvons simplement y lire: "Marie poignarde, encore et encore, le Roi... ${ }^{6}$ » Macaigne opère donc un glissement lorsqu'il passe à la scène, du sang à la peinture rouge et des excréments à la peinture noire. Dans En Manque, c'est d'ailleurs aussi à grands jets de peinture noire que la riche collectionneuse est mise à mort, lors du coup d'état révolutionnaire de sa fille. La peinture noire est ensuite projetée sur les tableaux qui sont tous détruits. Nous ne disposons pas du texte publié de la pièce, mais nous pouvons imaginer que, en reprenant l'image de la peinture noire déversée sur la tête d'une figure de pouvoir, Macaigne rejoue ce glissement entre peinture noire et excréments. La peinture vient donc remplacer les matières organiques, avec qui elle partage de nombreuses caractéristiques physiques : des proximités de teintes, le fait de choir ou de s'écouler, le fait de faire tache. 
fig. 1 : En Manque, Vincent Macaigne, Théâtre Vidy-Lausanne, Lausanne, Printemps 2017.

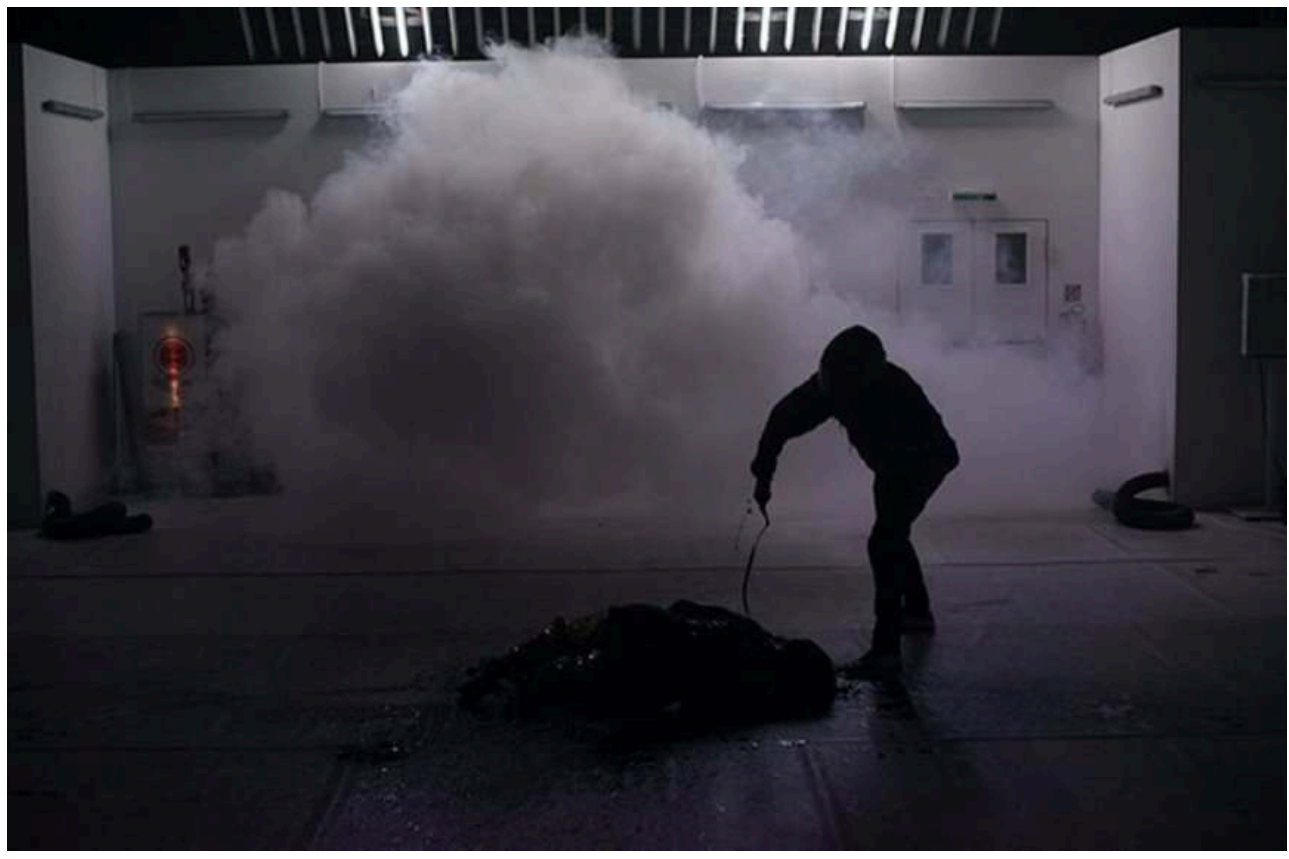

(c) Mathilda Olmi

12 Macaigne, qui orchestre dans ces deux spectacles des révolutions ratées ou trahies, joue en fait de cette peinture-matière de deux manières. Pour représenter le geste révolutionnaire, il joue sur la proximité de la peinture avec les humeurs. En ce sens, il intègre bien au sein de son écriture scénique des motifs repris aux artistes de performance des années 1970 - on pense en particulier aux Actionnistes Viennois, qui utilisaient dans leurs performances du sang (humain et animal) et des excréments. Pour ces artistes, il s'agissait de marquer leur refus de la société conservatrice d'alors, la libération des instincts humains les plus vils - qui allait avec la libération des humeurs du corps - réfléchissant cette fureur révolutionnaire. Macaigne réintègre cette libération de forces dans sa grammaire scénique; ces motifs empruntés aux Actionnistes deviennent les syntagmes d'une nouvelle écriture dont la peinture - qui remplace les matières organiques - devient l'encre. Ce passage des matières organiques à la peinture permet ensuite à Vincent Macaigne de rejouer cette fureur révolutionnaire, désormais « comme farce $»^{7}$.

13 Cette mise au premier plan de la peinture - qui apparaît sur scène comme peinture, dans des bouteilles et dans des seaux - est en effet aussi pour Macaigne une manière de souligner que tout ce qui se passe sur scène s'y passe pour de faux. Il fait ainsi dire à un de ses personnages, à l'ouverture de Je suis un pays, « N'ayez peur de rien, ici, rien de grave n'arrivera... ${ }^{8}$ 》 D'ailleurs, Marie a beau poignarder " encore et encore» le roi Immortel, celui-ci, comme son nom l'indique, ne meurt jamais. Toute cette profusion de peinture n'aura servi à rien. De même, la révolution de Marie tourne court; elle est assassinée par des policiers, et c'est le personnage le plus vil, pensé comme une caricature de Donald Trump, qui prend le pouvoir. Dans En Manque, la révolution est trahie par celles-là mêmes qui l'avaient perpétrée : alors qu'elles avaient juré de se tuer, une fois la révolution réussie, elles finissent par prendre le pouvoir, et décident de dédier les anciens lieux du pouvoir à la fête. La peinture, comme instrument du faux (faux sang, faux excréments), permet aux personnages macaigniens de jouer aux faux 
révolutionnaires. Plus ils en déversent, et plus leurs gestes perdent en précision. Ces révolutions manquées et leur lot de peinture ajoutent en effet au chaos de la scène, qui, dans les deux spectacles, est également envahie peu à peu d'autres matières : terre, eau, fumée, etc. Macaigne retourne donc la peinture à sa forme la plus élémentaire : de la peinture cadrée avant que les tableaux ne soient détruits, à la peinture déversée en signe de révolution, jusqu'à la peinture comme matière première d'une scène en ruines.

Il y a en effet dans la dramaturgie macaignienne un appel vers le non-sens, toujours empêché par une volonté contraire de signifier, voire de faire passer un message. C'est en ce sens qu'il faut comprendre les harangues finales qui presque toujours concluent ses spectacles; une figure (souvent porte-parole de l'auteur) tire les conclusions de l'histoire qui vient de se jouer, et appelle les spectateurs à faire mieux, et le plus souvent, à réapprendre à s'aimer. Cette parole très construite est cependant prise dans un double phénomène d'hystérisation (en ce sens qu'elle devient elle-même le signe d'une émotion imprononçable) : elle est hurlée (souvent dans un mégaphone), et elle arrive au milieu des décombres et de la peinture. Cette tension entre parole construite et matière informe associée au cri rejoue la tension déjà évoquée entre tableau cadré et peinture-matière : la fable macaignienne oscille ainsi constamment entre recherche de sens et sidération, tension qui amène le hurlement.

\section{"Sauver le Moyen-Age de la Renaissance ", ou la pratique résistante de Jan Fabre}

15 Chez Jan Fabre, qui lui aussi exploite le potentiel subversif de l'écoulement de peinture, les matières liquides (eau, urine, faux sang, sueur...) précèdent l'arrivée de la peinture sur le plateau, et semblent même la préparer. Le metteur en scène rêve ainsi l'écoulement de peinture, de Je suis Sang (2001) à L'Histoire des Larmes (2005), avant de le rendre véritablement concret dans l'une de ses dernières créations pour la scène: Mount Olympus (2017). C'est en faisant retour à L'Histoire des Larmes, créée dans la Cour d'honneur du Palais des Papes, à Avignon, que nous pouvons comprendre ce que ce fantasme de l'écoulement contient de révolte.

Sur un plateau presque nu, un Diogène canin parcourt l'espace à la recherche d'un homme, alors que notre époque policée a renoncé aux odeurs. L'Histoire des Larmes veut redonner sa place à cette eau qui nous constitue en majorité et que nous tentons pourtant par tous les moyens de dissimuler : larmes, mais aussi urine et transpiration, Fabre met en fête ce qui doit normalement rester caché. Il s'agit, proclame le chevalier du désespoir, autre personnage de cette grande fresque moyenâgeuse, de «sauver le Moyen-Age de la Renaissance » : refaire place au corps suintant, liquide, transpirant, désormais analysé, répertorié et mis en cases sous l'œil omniscient des sciences de la Renaissance.

17 Il peut être intéressant de noter que la peinture de Rogier Van der Weyden est une des références picturales les plus importantes de la pièce. Fabre revendique en effet son attrait pour la peinture flamande, dans laquelle il puise beaucoup de ses sources d'inspiration. Dans L'Histoire des Larmes, il dessine ainsi avec le corps de ses performeurs des descentes de croix et des Pietà directement inspirés de certains tableaux de Van der Weyden : 
Fig. 2 : L'Histoire des Larmes, Jan Fabre, Cour d'honneur du Palais des Papes, Juillet 2005.

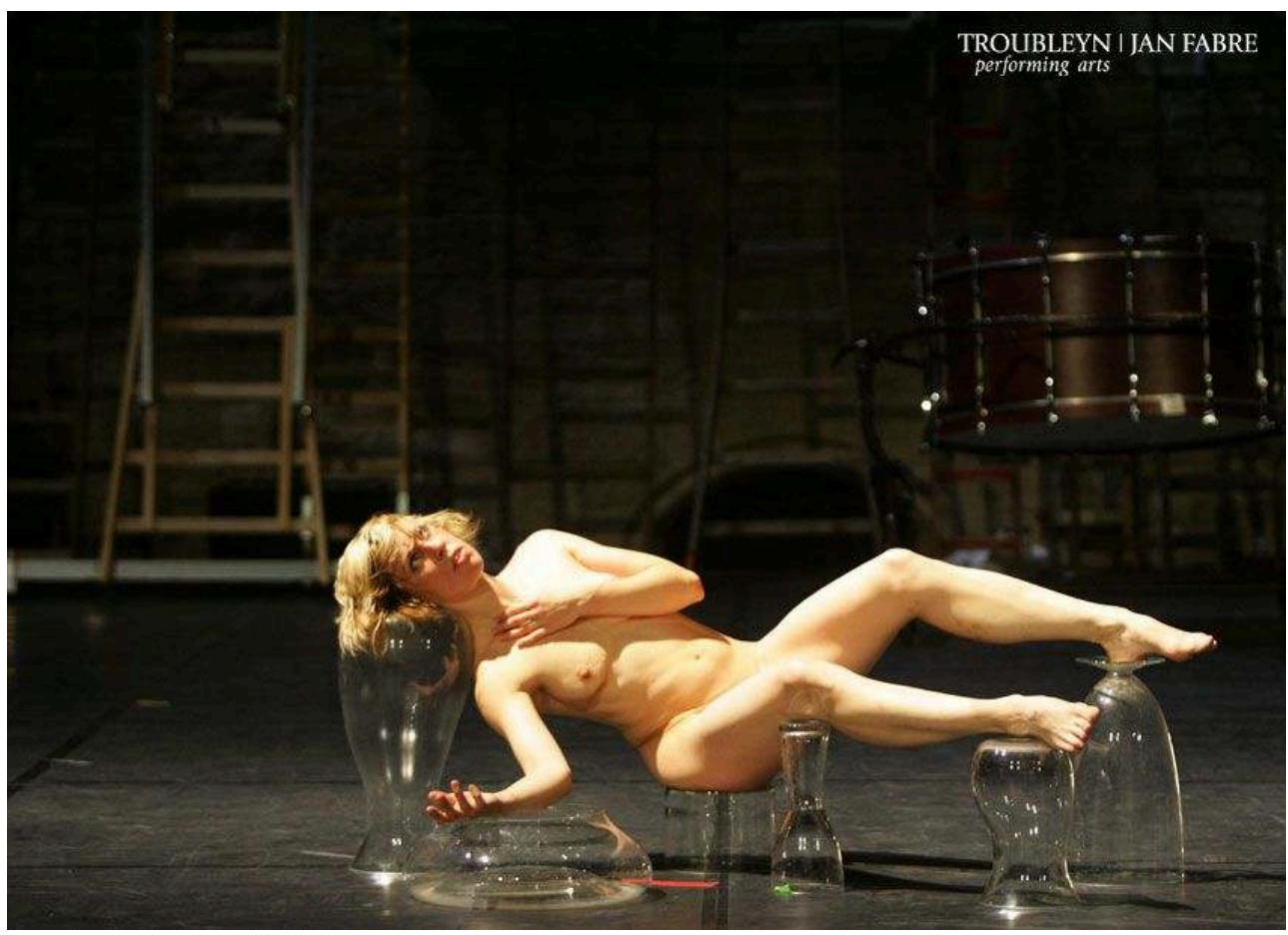

(c) Wonge Bergmann

Fig. 3 : Pietà, Atelier de Rogier Van der Weyden.

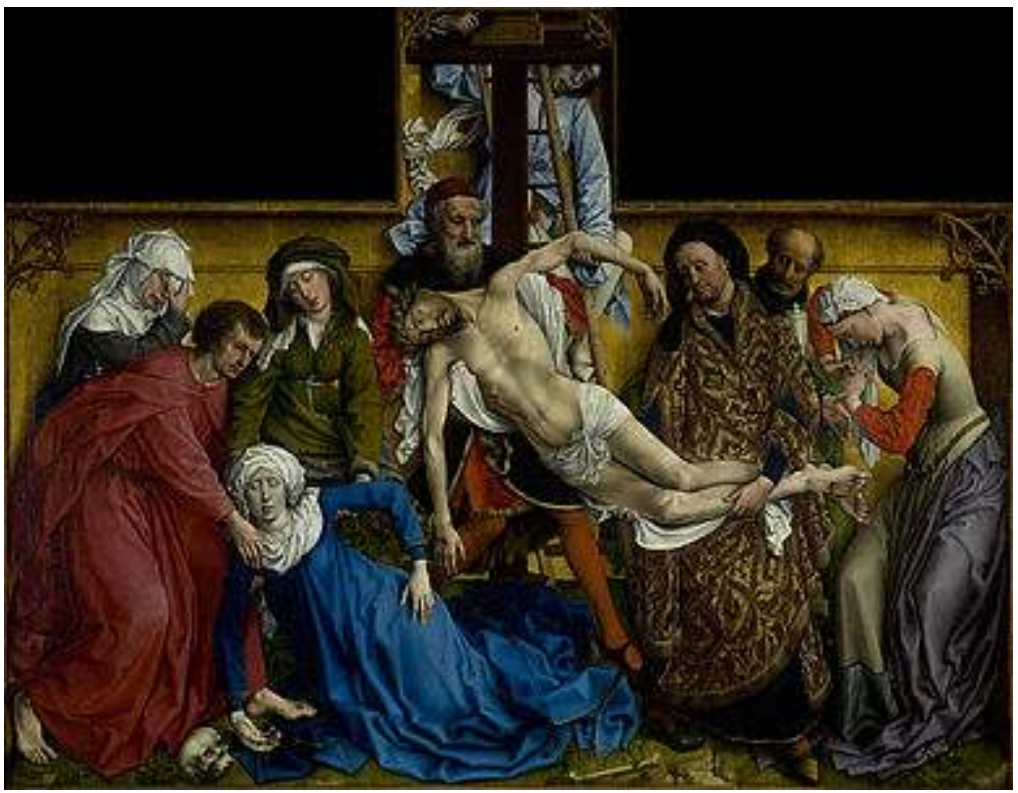

Vers 1465, 35,5 x 45 cm, National Gallery, Londres.

18 Or, Rogier Van der Weyden est justement un peintre qui travaille l'écoulement. Voici ce que l'historien de l'art Guillaume Cassegrain écrit à propos d'une Crucifixion du peintre :

Ce que Van der Weyden avait inventé afin d'accompagner et d'intensifier la déchéance de la mort ne tenait pas exclusivement à l'iconographie (la lamentation de saint Jean et de la Vierge) ou à la figuration (les larmes, notamment) mais aussi [...] à l'invention formelle, à l'expressivité de la peinture en soi. Les longues 
coulures du mur que Rogier a peintes avec une très grande méticulosité donnent à voir l'écoulement général, l'affliction qui affecte la totalité du monde visible. Les murs suintent et expriment par là, phénoménologiquement, de façon "pathique ", ce que ressentent les figures sacrées de la Crucifixion'. peinture apparaît progressivement, par déversements successifs, avant d'être épandue à flots sur la scène et les performeurs, lors des célébrations orgiaques de la fin du spectacle.

21 Au cours de la performance, les premiers jets de peinture sont associés au sang et au sperme. Ainsi, avant que des jaillissements de peinture de toutes les couleurs n'envahissent le plateau lors du dernier chapitre, la peinture qui s'écoule à grands jets est soit rouge, soit blanche. On note sa première véritable apparition lors d'un épisode intitulé « Pornè's dream about the traveling wig » («Le rêve de Pornè sur la perruque itinérante »), lors duquel certains performeurs qui miment divers sacrifices déversent des bouteilles de peinture rouge sur d'autres performeurs, figures des sacrifiés. Ce premier jaillissement de peinture est, dans le titre de l'épisode, associé à la figure de Pornè (étymologiquement la prostituée, mais qui renvoie aussi à la question de l'obscène, le pornographe étant précisément celui qui montre ce qui normalement doit rester caché). Or, lors de la scène précédente, la performeuse jouant Pornè avait mimé une très longue scène de fellation. Jan Fabre orchestre une montée de tension au cours de cette première scène, le moment de l'éjaculation n'arrivant symboliquement que lors de la scène suivante, celle du "rêve de Pornè ", où le sang se met soudain à jaillir. Ce lien est ensuite rejoué dans les deux épisodes intitulés "women spoiled by the gods " (les femmes gâtées par les dieux) et «men spoiled by the gods» (les hommes gâtés par les dieux), où l'on voit des performeurs errer sur la scène, couverts cette fois d'une épaisse peinture blanche, dont ils se sont recouverts en coulisses. Ici le terme « spoil » est polysémique, tout comme le terme "gâter » en français. Fabre renvoie d'un côté à la jouissance - l'image serait celle d'humains fécondés par les dieux, les coulures de peinture blanche renvoyant à l'idée d'un sperme divin. Mais ce sont aussi des êtres "gâtés" ou "gâchés » qui apparaissent sur scène, essayant de se défaire frénétiquement de la peinture dont ils ont été recouverts (le viol des humains par les dieux étant un thème très présent dans la mythologie grecque, d'Europe à Proserpine...). 
Fig. 4 : Mount Olympus, Jan Fabre, Skirball Center, New York, 2018.

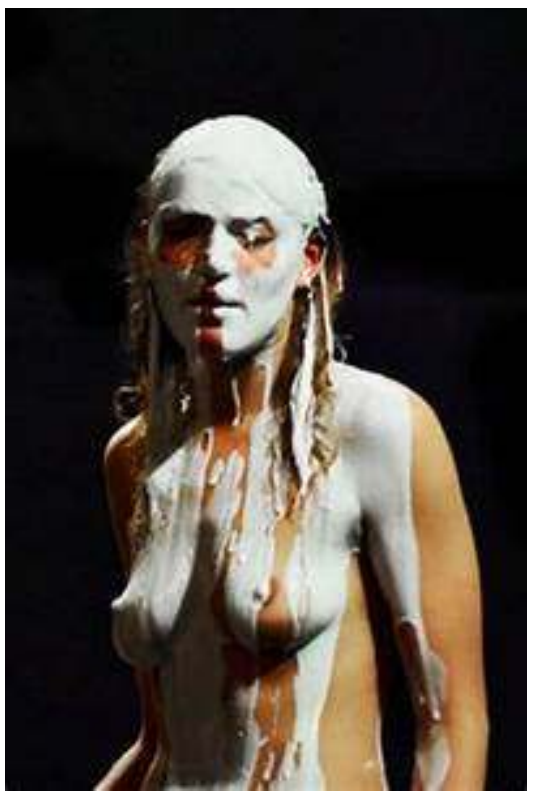

(c) Sam de $\mathrm{Mol}$

L'écoulement de peinture arrive donc sur la scène fabrienne comme signe des pulsions humaines: désir sexuel et désir de mort. Le sous-titre choisi par Fabre pour son spectacle, « to glorify the cult of tragedy » (pour glorifier le culte de la tragédie), est en cela significatif. Comme le rappelle Hans-Thies Lehmann dans le texte qui accompagne la présentation du spectacle, «la tragédie a essentiellement à voir avec la transgression, elle est par excellence le théâtre où l'on expérimente comment les normes sociales, les lois comportementales et les règles morales peuvent être profondément mises à mal ${ }^{10}$. » Fabre fait d'ailleurs de Dionysos une figure centrale de son spectacle, comme symbole d'une affirmation radicale de vie. Sur le plateau, ce Dionysos est double, à la fois mâle et femelle, interprété par un acteur et une actrice obèses dont les silhouettes rappellent le Silène de Van Dyck. C'est bien sûr La Naissance de la Tragédie que le metteur en scène a à l'esprit lorsqu'il crée cette double figure dionysiaque. Alors que nous vivons selon Fabre dans une époque où l'apollinien (c'està-dire l'ordre et la mesure) a tout envahi, il s'agit de libérer à nouveau les forces chaotiques du dionysiaque.

Avec ces explosions de peinture - des premières éjaculations au jaillissement final, lors duquel les performers se déversent à grand jets, les uns sur les autres, des peintures de tous les couleurs -, Fabre revient à son obsession : le refus du corps d'être enchainé par l'esprit, et la nécessité de faire ressortir à la surface ce qui est d'ordinaire réprimé. S'il y a purgation dans la tragédie fabrienne, c'est donc une purgation du corps, dans une grande fête orgiaque et carnavalesque où le dedans retrouve le dehors (parmi les matières qui jonchent la scène à la fin du spectacle, on trouve ainsi des abats d'animaux, qui donnent à voir l'intérieur du corps). Il s'agit pour Fabre d'effacer toutes les frontières, tout ce qui d'ordinaire limite les corps. Dans cette perspective, la peinture vient rejouer l'écoulement du corps suintant, sécrétant, pour un retour à un monde où tout s'interpénètrerait, bien loin de l'idéal classificateur de l'humanisme renaissant. 


\section{Souillure et défi chez Romeo Castellucci}

scène de l'opéra de Schönberg, Moses und Aron, créé à l'Opéra de Paris en 2015. Cette peinture apparaît alors que les Hébreux doutent de la révélation que Moïse leur apporte, d'un Dieu unique et invisible. Lors de la prédication de Moïse, elle s'écoule du plafond sur la main du prophète, et à sa vue, Aron s'exclame : "Voyez ? La lèpre ! ». Doute et maladie sont donc assimilés, et la peinture noire est d'abord le signe de ce doute qui peut devenir infectieux. Lors de la deuxième partie de l'opéra, alors que Moïse s'est retiré sur le Mont Sinaï pour recevoir les tables de la Loi, les Hébreux recommencent à douter de sa parole, et construisent un veau d'or qu'ils pourront adorer. Castellucci place alors un bassin de peinture noire au centre de la scène; pendant tout l'épisode du veau d'or, les membres du peuple hébreu ayant abjuré leur foi en Moïse et en un Dieu unique et invisible iront s'y immerger et en ressortiront couverts de noir. Aron, qui a autorisé la confection du veau d'or, est lui aussi recouvert de peinture (par deux performers armés de bidons). Finalement, ceux qui tentent de rester fidèles à Moïse sont sacrifiés, dans une cérémonie lors de laquelle des performers déversent à nouveau sur eux des bidons entiers de peinture noire :

Fig. 5 : Moses und Aron, Romeo Castellucci, Opéra de Paris, Paris, 2015.

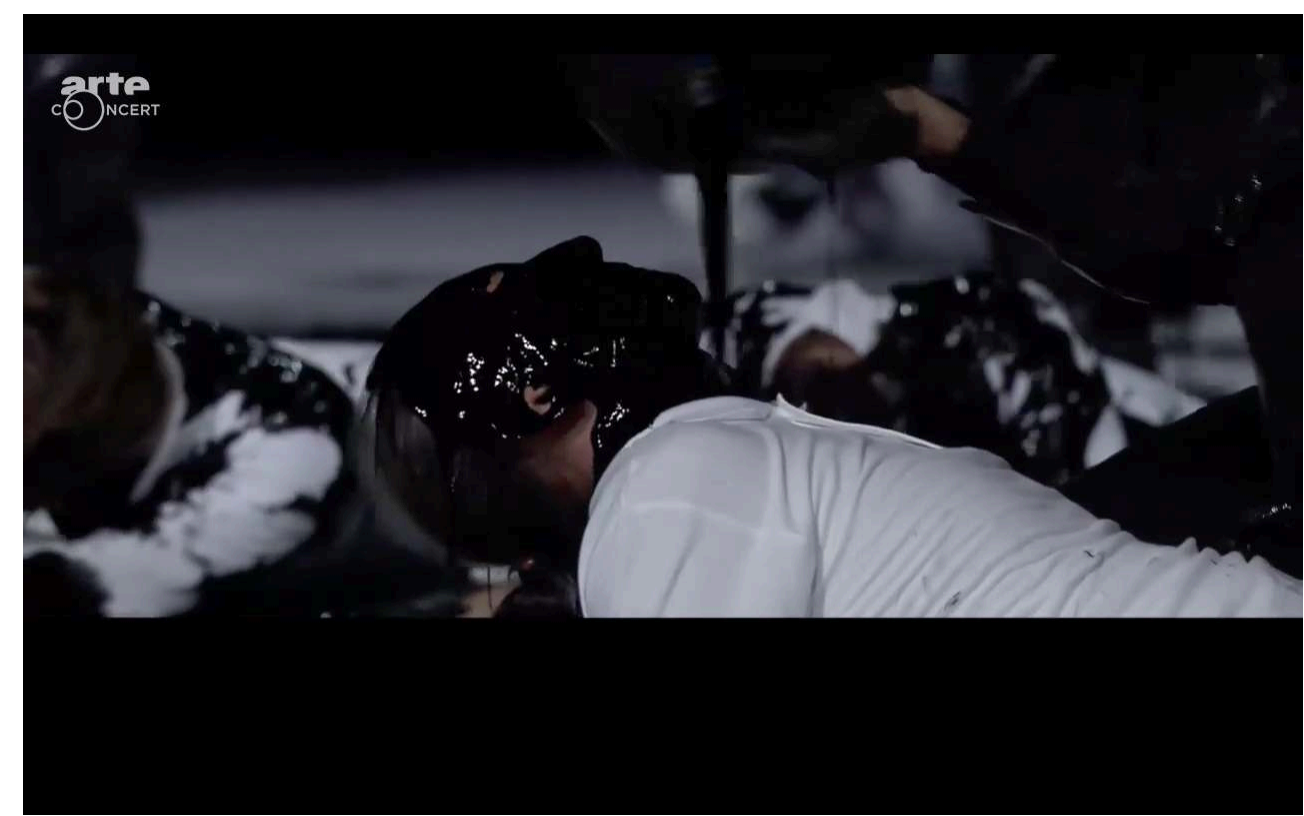

(C) Droits réservés

Castellucci utilise donc à plein le potentiel de cette peinture matière, qui, dans son essentielle fluidité, peut recouvrir différentes significations, ou plutôt passer d'un sens à un autre en s'enrichissant de toujours plus de significations. Ainsi, alors que la peinture noire, dans la première partie, symbolisait le doute qui s'insinuait dans l'esprit du peuple hébreu au moment où Moïse leur révèle l'existence d'un Dieu unique et irreprésentable, elle en vient, lors de la deuxième partie, à représenter une autre forme 
de croyance, en des divinités multiples et païennes, et surtout palpables et représentables, qu'on impose aux tenants d'un Dieu unique (lors de la cérémonie où ils sont de force aspergés de peinture). C'est alors le doute même qui fournit le fondement de cette croyance renouvelée dans les idoles, ce que Castellucci souligne en les représentant par le même élément. Le metteur en scène montre ainsi comment le doute, qui est selon lui le noyau de la foi ${ }^{11}$, peut aussi très facilement se retourner contre elle, et nous ramener à des croyances idolâtres.

Si l'on adopte le point de vue de Moïse et de l'Ancien Testament, la peinture fait donc signe vers la souillure du peuple qui a douté, et qui, pour cette raison, subira la colère de Dieu. Castellucci renforce cette impression en faisant contraster la scène et les costumes immaculés (ceux du peuple élu) et cette peinture d'un noir de jais qui envahit peu à peu le plateau. Si l'on adopte en revanche le point de vue d'Aron, qui ne cesse de répéter à Moïse que les êtres humains sont des êtres sensibles, qui ont besoin de sentir et de toucher, d'éprouver les idées pour les comprendre, la peinture change de sens et devient l'arme d'un peuple en révolte contre la parole tyrannique de Moïse, qui prêche l'invisible. La peinture, si elle est le symbole du crime perpétré par les Hébreux, semble en effet arriver presque comme un soulagement sur la scène immaculée : c'est au moment où elle a envahi la scène que Castellucci fait intervenir des performeursdanseurs, qui s'y donnent corps et âmes, communiquant par là-même cette impression de jouissance enfantine au public. Castellucci réussit donc ce tour de force, en jouant sur les capacités capillaires de la peinture, d'utiliser la même matière comme symbole et comme indice ${ }^{12}$. La peinture noire est en effet à la fois le symbole du doute qui envahit le peuple hébreu, mais elle fonctionne aussi comme indice de la capacité créatrice de ce même peuple (le peuple qui a façonné le veau d'or), à partir du moment où ils se mettent à l'utiliser comme peinture dans leur cérémonie de baptême païenne. À la fin du spectacle en effet, il ne reste que les traces de cette peinture sur le drap blanc qui recouvre la scène, trace de l'activité créatrice qui a eu lieu sur scène, sous nos yeux.

Le metteur en scène joue d'ailleurs à nouveau de cette capacité de la peinture à glisser d'une signification à une autre à la fin du spectacle. Aron, alors qu'il comprend et reconnaît véritablement l'existence du Dieu éternel, déverse sur tout son corps de la peinture dorée, qui se trouve dans deux cornes d'animal que le chanteur interprétant Moïse a apportées avec lui sur le plateau, en revenant du Mont Sinaï. Romeo Castellucci s'amuse ici d'une erreur de traduction qui a perduré des siècles durant et est à l'origine des plus grands chefs-d'œuvre de la peinture et de la sculpture occidentales (en particulier le Moïse de Michel-Ange). Il est en effet écrit dans le Bible qu'alors que Moïse redescend du Mont Sinaï, la peau de son visage émet de la lumière ; on a longtemps cru cependant que ces traits de lumière qui sortaient du front de Moïse étaient des cornes. La peinture dorée contenue dans les cornes symbolise alors cette lumière, elle-même indice de l'esprit divin. Dans de nombreuses Annonciations, le Saint Esprit est ainsi représenté par un trait de lumière qui tombe sur Marie, alors qu'elle écoute les mots de l'Ange. 
Fig. 6 : L'Annonciation, Fra Angelico.

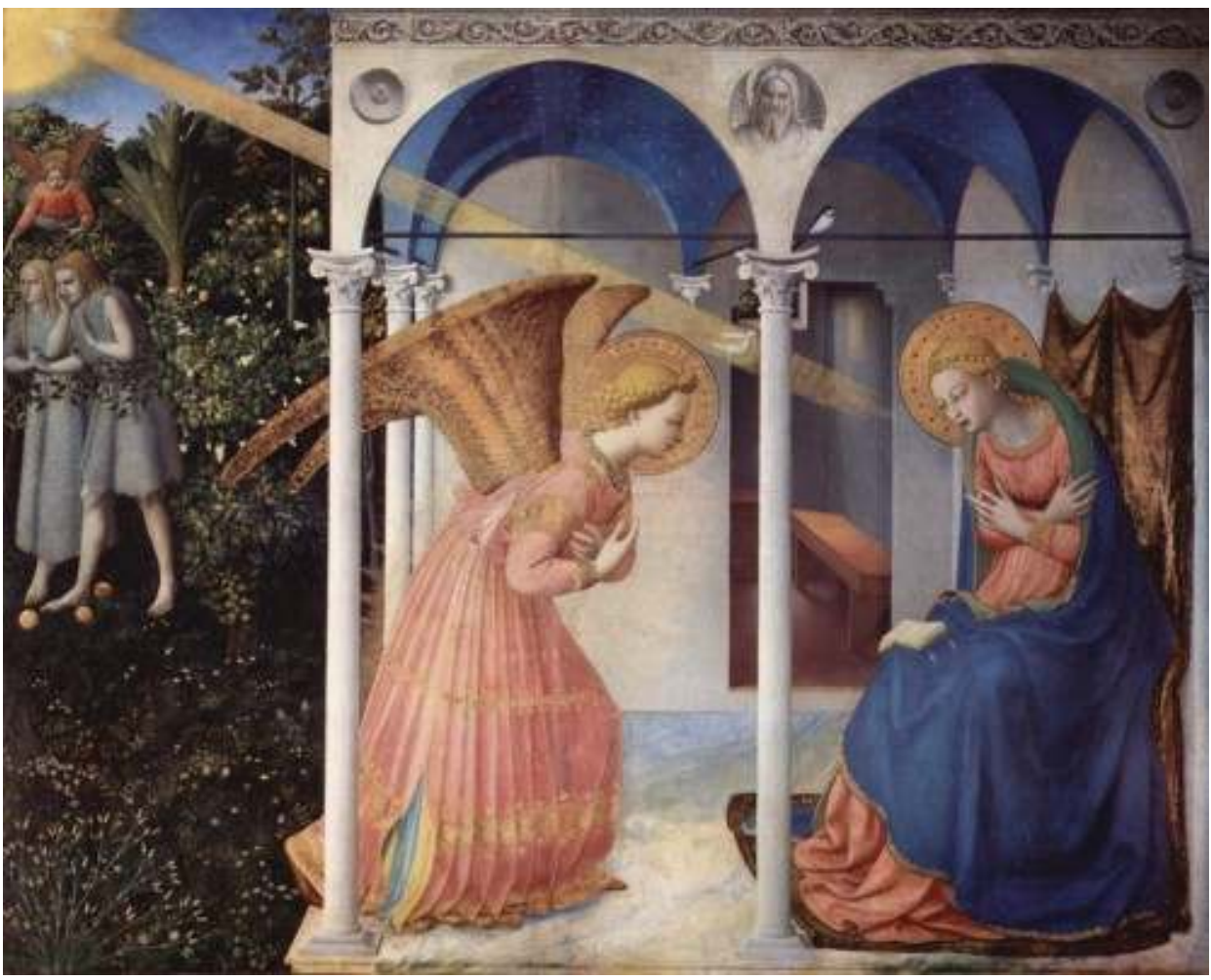

vers 1430, 194 x 194 cm, musée du Prado, Madrid.

29 Cette image qui arrive à la fin du spectacle annonce ainsi également le moment de l'Incarnation, qui résoudra le problème soulevé par l'opéra (à savoir la nécessité pour les êtres humains d'éprouver les idées dans leur chair face à un Dieu invisible et irreprésentable).

30 On remarque donc qu'il y a chez nos trois metteurs en scène une tension - plus ou moins consciemment utilisée par les uns et les autres - entre la volonté d'utiliser la peinture comme symbole et la volonté d'utiliser les caractéristiques propres à la peinture, en particulier sa capacité à déborder le langage articulé. Si l'on reprend la tripartition peircienne déjà évoquée, on pourrait même dire que cette peinture-matière qui se déverse sur scène le fait à la fois comme indice, symbole, et icône ${ }^{13}$. La peinture, lorsqu'elle est utilisée en place des excréments ou des humeurs corporelles (nous l'avons vu dans les spectacles de Fabre et de Macaigne, mais c'est aussi le cas dans un spectacle de Castellucci comme Sur le concept du visage du fils de Dieu), semble s'en faire l'icône : elle partage en effet avec eux de nombreuses caractéristiques (la couleur, la liquidité, le fait de choir et de faire tache), et surtout, il est parfois difficile pour les spectateurs de discerner la nature de l'élément qui s'écoule sous leurs yeux. Ainsi, dans Sur le concept du visage du Fils de Dieu (2011) - spectacle lors duquel un vieil homme incontinent était pris de crises de diarrhée à répétitions -, Romeo Castellucci ajoute à la représentation des crises une odeur d'excréments, et travaille la peinture brunâtre pour en faire une matière assez épaisse. La ressemblance est alors poussée à l'extrême, et l'on finit par confondre l'objet et sa représentation: il s'agit bien d'un fonctionnement iconique. Un signe est un symbole lorsqu'il renvoie à son objet en vertu d'une convention, ou en tout cas d'un accord préalable: la peinture est donc symbole quand elle représente, tour à tour, le geste de révolte chez Macaigne, le signe 
de la résistance au cadrage scientifique chez Fabre, et le doute chez Castellucci. Enfin, un signe renvoie à son objet de manière indicielle lorsqu'il entretient un rapport de contiguïté matérielle avec l'objet ; c'est le cas par exemple lorsque la peinture devient la trace de l'activité artistique du peuple hébreu dans Moses une Aron.

31 À cela, il faut ajouter qu'à plusieurs reprises, la peinture sur scène ne renvoie plus qu'à elle-même, comme matière informe et chaotique - ainsi des scènes finales de Vincent Macaigne. C'est cette dernière utilisation de la peinture, qui est liée à une interrogation sur la création en même temps que sur nos origines, que nous nous proposons à présent d'étudier.

\section{Voyage de la matière, voyage dans la matière : Chaos, Genèse et Déluge}

\section{Les récits fondateurs de Romeo Castellucci : versement et sacrifice}

Ce travail sur les origines est particulièrement apparent dans le théatre de Romeo Castellucci, qui a entrepris depuis plusieurs années une exploration des grands récits bibliques, de Inferno. Purgatorio. Paradiso (2008) à Moses und Aron (2015), en passant par Sur le concept du visage du Fils de Dieu (2011). Il orchestre en fait ce retour à des préoccupations d'ordre théologique dès sa Tragedia Endogonidia (2002-2004), spectacle divisé en onze épisodes (présentés dans dix villes européennes différentes), et qui rejoue en son sein l'advenue du Christ (qui arrive lors de l'épisode 6). Les épisodes 4 et 5 mettent en jeu des figures de martyrs ; on peut y voir tour à tour un homme juif passé à tabac (à grands jets de peinture rouge), et une petite fille tout droit sortie des Souliers Rouges d'Andersen, qui subit, les bras en croix, un étrange baptême au sang (là aussi de la peinture rouge), préfigurant ainsi l'advenue du Christ. 
Fig. 7 : Tragedia Endogonidia, B \#04 Bruxelles, Romeo Castellucci, Bruxelles, 2003.

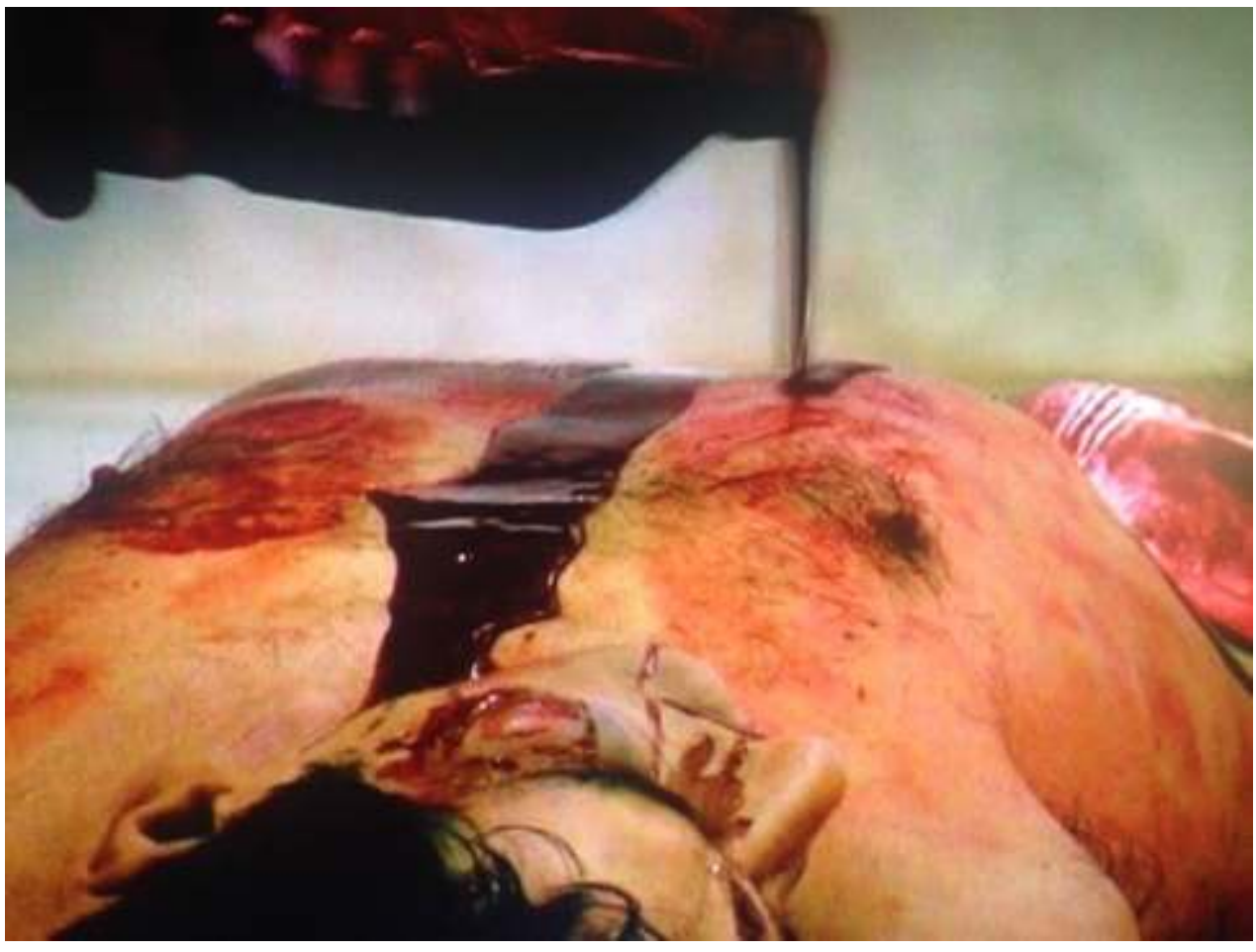

(c) Droits réservés

Fig. 8 : Tragedia Endogonidia, BN \#05 Bergen, Romeo Castellucci, Bergen, 2003.

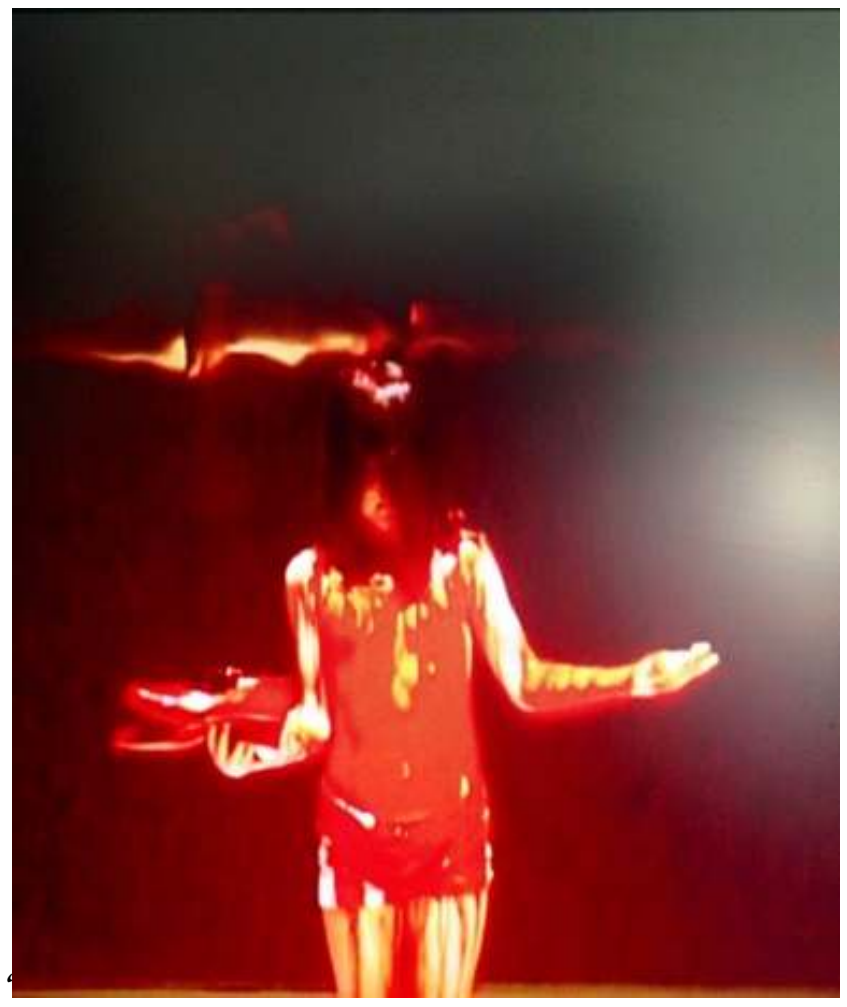

(c) Droits réservés 
Enfin, l'épisode 6 constitue une transition entre l'Ancien et le Nouveau Testament. Il s'ouvre sur une recréation du sacrifice d'Abraham (toujours à grand renfort de peinture rouge), avant l'arrivée sur la scène d'une figure christique, les reins ceints d'un linge blanc et coiffé d'une couronne d'épines.

Fig. 9 et 10 : Tragedia Endogonidia, P\#06 Paris, Romeo Castellucci, Paris, 2003.
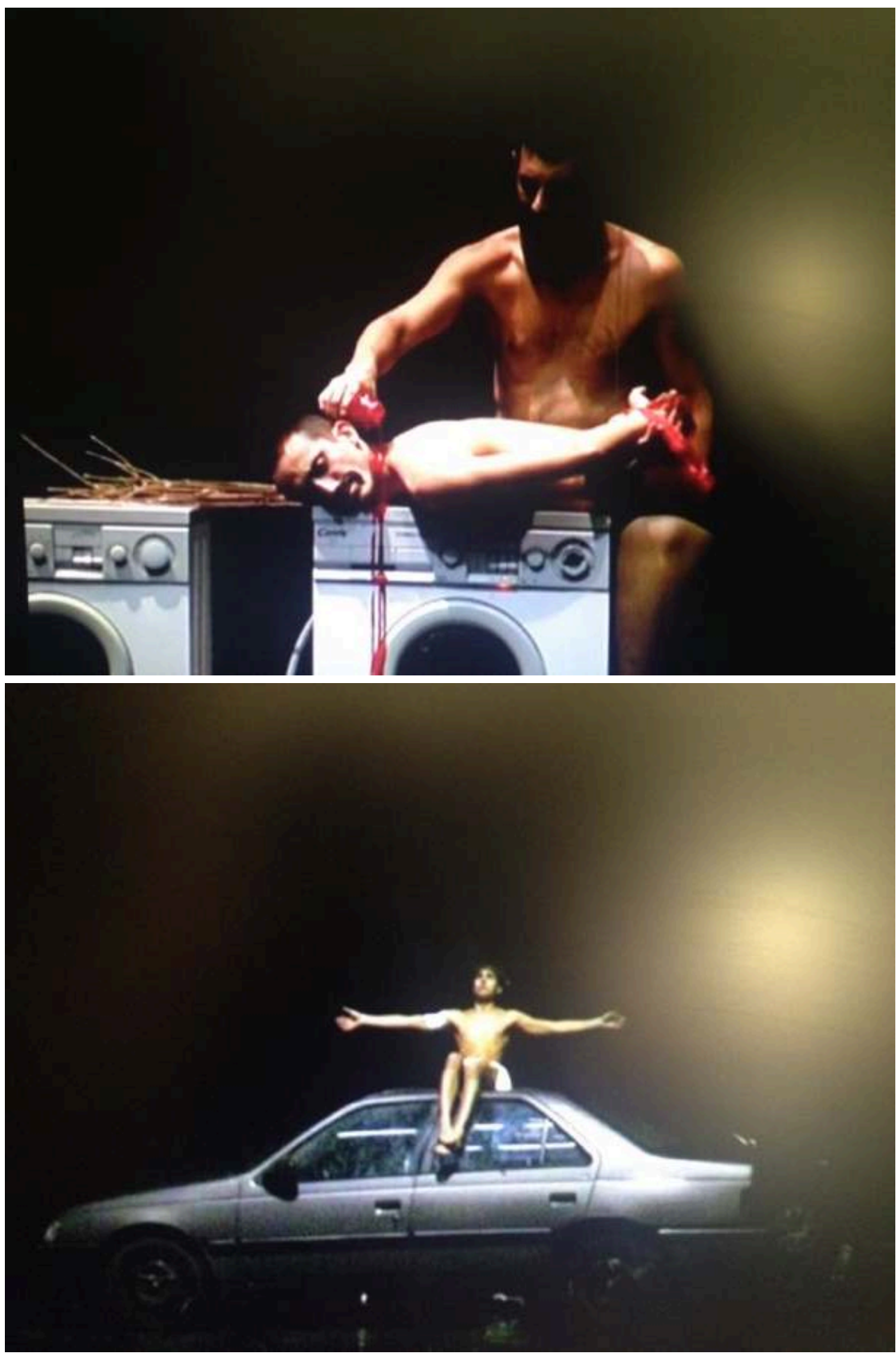

(c) Droits réservés 


\section{peinture comme symbole et l'utilisation de l'écoulement de la peinture pour lui-même,} ici comme phénoménologie du sacrifice christique. Nous nous trouvons au cœur de la mythologie castellucienne: le metteur en scène n'a en effet de cesse d'entrelacer réflexion théâtrale (qui est toujours, chez lui, une réflexion sur la tragédie, d'où l'importance du sacrifice) et théologie chrétienne. Dans la Tragedia Endogonidia, il part ainsi du sacrifice comme acte profondément tragique et inévitable, pour arriver à l'incarnation et à la résurrection. Il faut bien sûr souligner que ce moment de l'incarnation/résurrection arrive toujours, sur la scène de Castellucci, pour être mis en question. On pense ainsi aux mots qui apparaissent à la fin de Sur le concept du visage $d u$ fils de Dieu, "You are (not) my shepherd» (tu (n') es (pas) mon berger), adressés au visage déchu du Christ, ou encore aux nombreuses torsions qu'il fait subir aux images de l'Annonciation dans Il Primo Omicidio (oratorio de Scarlatti qu'il présente à l'opéra de Paris en janvier 2019). Dans la Tragedia Endogonidia, on remarque que c'est justement lorsque le Christ arrive sur scène que Castellucci cesse de faire couler la peinture rouge. Il rompt ainsi le lien entre versement et sacrifice qu'il avait instauré, refusant au Christ ses sanies. Castellucci, en nous ramenant aux origines des récits qui nous fondent, et en explorant ces figures de martyrs et de sacrifiés qui les peuplent, fait ainsi du sang versé ce qui doit nous sauver, en empruntant à l'imaginaire chrétien, tout en le déplaçant, puisque le sauveur n'est plus celui de la tradition. Si le versement de peinture, sur scène, rejoue l'image du sacrifice originel, c'est désormais l'acteur, en montant sur le plateau, qui se sacrifie pour nous ${ }^{15}$. Castellucci rejoue ainsi ce lien entre écoulement et sacrifice, tout en le laïcisant.

L'image du sang versé n'explique cependant qu'en partie l'arrivée à flots de la peinture qui, dans certains spectacles (tels Je suis Sang de Fabre, ou En Manque de Macaigne), envahit complètement le plateau, dans une recréation de deux épisodes bibliques bien plus anciens que celui de l'Incarnation : la Création du monde et le Déluge.

\section{Macaigne, Fabre et le Déluge}

Dans En Manque, Vincent Macaigne place dans les cintres une bâche qui semble pleine de liquide. Au cours du spectacle, cette bâche devient de plus en plus lourde à mesure qu'elle se remplit, et s'enfonce dangereusement vers la scène en son milieu. Enfin, la bâche cède et le plateau est envahi d'une eau noire qui vient faire écho à la peinture 
noire déjà utilisée pour la destruction des tableaux du Caravage, lors de l'épisode révolutionnaire ${ }^{16}$. Cette image d'une destruction par les eaux venues du ciel nous ramène alors à l'épisode du Déluge.

Fig. 11 : En Manque, Vincent Macaigne, Théâtre Vidy-Lausanne, Lausanne, 2017.

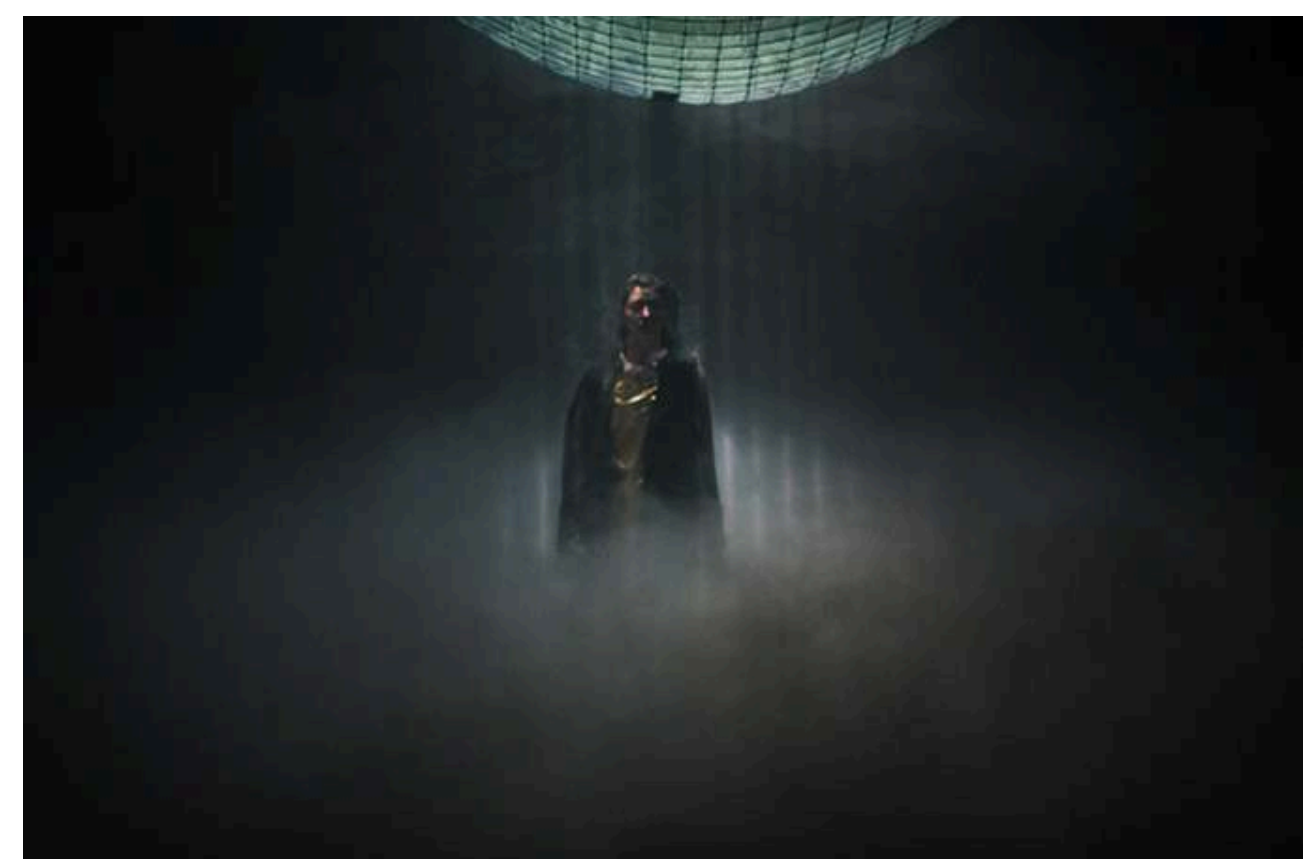

(C) Mathilda Olmi

Fig. 12 : En Manque, Vincent Macaigne, Théâtre Vidy-Lausanne, Lausanne, 2017.

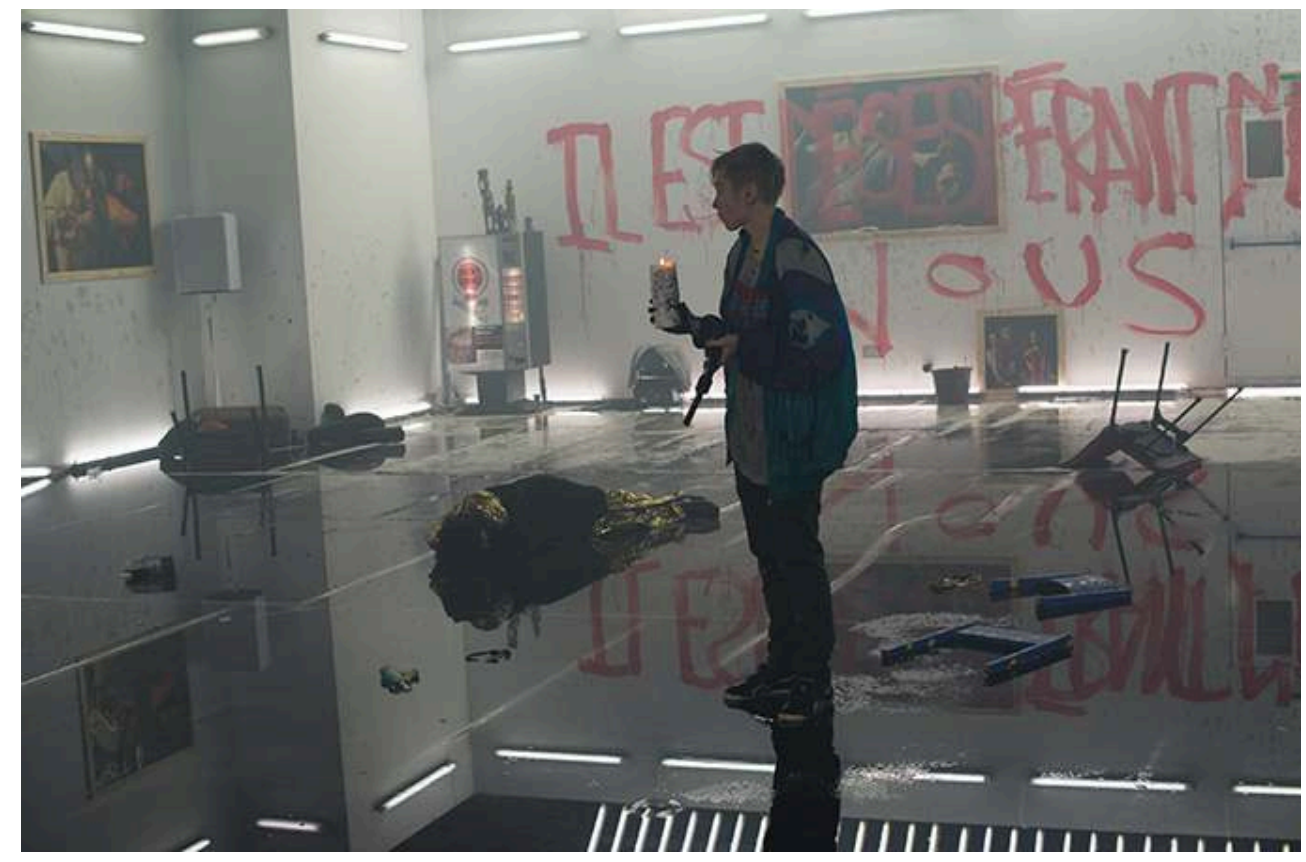

(C) Mathilda Olmi

39 Ce plateau envahi par les eaux semble renvoyer à la volonté affichée par Macaigne de faire table rase du passé, et s'inscrit dans la même logique que celle de la destruction 
des chefs-d'œuvre. Le metteur en scène, en composant cette fable apocalyptique, demande en fait à plusieurs reprises - par l'intermédiaire de ses acteurs - comment il est encore possible d'inventer de nouvelles histoires, et de faire œuvre originale, alors que tout a déjà été dit. Le Déluge arrive comme une solution à cet empêchement créateur, dans un geste semblable au geste préconisé par Artaud dans « Le Théâtre et la culture » :

On peut brûler la bibliothèque d'Alexandrie. Au-dessus et en dehors des papyrus, il $\mathrm{y}$ a des forces: on nous enlèvera pour quelque temps la faculté de retrouver ces forces, on ne supprimera pas leur énergie. Et il est bon que de trop grandes facilités disparaissent et que des formes tombent en oubli, et la culture sans espace ni temps et que détient notre capacité nerveuse reparaîtra avec une énergie accrue. Et il est juste que de temps en temps des cataclysmes se produisent qui nous incitent à en revenir à la nature, c'est-à-dire à retrouver la vie ${ }^{17}$.

Le spectacle poursuit d'ailleurs la réflexion artaudienne en proposant une variation sur la copie et le retour du même. Si Sofia Burini, la riche collectionneuse de l'histoire, décide de donner accès à une partie de sa collection de chefs-d'œuvre de l'art occidental, elle fait finalement appel à un faussaire, Ulrich von Sidow, et ce sont des copies des tableaux qui sont exposées, alors que les originaux sont gardés dans des coffres dissimulés derrière les tableaux. La fable inventée par Macaigne est aussi une variation sur La Montagne Magique, de Thomas Mann. Son spectacle raconte l'histoire des "gens d'en haut", qui sur leur Mont Olympe, se sont recréé une vie autour des chefs-d'œuvre de la culture, et étouffent dans la répétition du même, loin des gens de la vallée. Comme le héros de Thomas Mann, Hans Castorp, finit par comprendre que la maladie et la mort sont un passage nécessaire pour accéder à la connaissance et à la vie, Sofia Burini finit par reconnaître la nécessité de sa propre mort et de la destruction des tableaux, afin de libérer les forces vives de l'art ${ }^{18}$.

41 Il faudrait également faire un sort au retour, dans le spectacle, d'un imaginaire issu du performance art des années 1970. En Manque est ainsi présenté comme "une étude théâtrale, plastique et chorégraphique pour acteurs, danseurs, et circassiens $»^{19}$. La scène finale, où les acteurs se roulent deux à deux dans la peinture, ne peut manquer de faire penser à la performance de Carolee Schneemann intitulée Meat Joy (1964), où des performeurs se roulaient ensemble dans de la peinture et des cadavres d'animaux, œuvre décrite par Schneemann elle-même comme un rite érotique et excessif.

42 C'est à cette quasi-impossibilité de s'affranchir de ce qui a déjà été fait - du chefd'œuvre de Thomas Mann aux performances des années 1970 - qu'il faut donc tenter d'échapper. Macaigne reproduit alors le schéma biblique du Déluge. Après une première création, et alors que le monde s'est essoufflé, le Déluge rédempteur arrive, et fait place à un chaos d'où pourra naître un nouveau monde que le personnage principal du spectacle, Liza, appelle de ses vœux dans son discours final, où elle enjoint aux spectateurs de rebâtir un monde fondé sur l'amour.

43 C'est aussi l'image théologique du Déluge que l'on retrouve sur les plateaux de Jan Fabre. À la fin de ses deux fresques moyenâgeuses ${ }^{20}$ présentées dans la Cour d'Honneur du Palais des Papes, le plateau est envahi de liquide. Dans Je suis Sang (2001), la peinture rouge s'immisce peu à peu par les failles d'un mur de fortune construit avec les praticables du spectacle, jusqu'à envahir tout à fait le plateau. Dans L'Histoire des Larmes (2005), l'image diluvienne est rejouée, alors que l'eau se met à couler à verse sur le plateau, comme venue du ciel. Mais le passage à Mount Olympus (2015) est peut-être ce qui nous permet de comprendre le mieux cette utilisation par les metteurs en scène de 
l'image diluvienne. Mount Olympus se termine en effet par une célébration orgiaque, où les performeurs se mettent à déverser sur scène, de manière anarchique, de la peinture de toutes les couleurs, et des confettis, également multicolores, alors que d'autres se lancent dans un «twerk » endiablé (l'épisode est intitulé "Danse rituelle extatique II (twerk final) »), au milieu des abats d'animaux et de la terre qui ont peu à peu recouvert le plateau au cours de la performance. Du déluge, on passe au chaos, dans cet enchevêtrement de matières où tout finit par se mélanger. C'est alors que les performeurs s'avancent face aux spectateurs, et, en une dernière incantation, les enjoignent à imaginer quelque chose de neuf: «Breathe, juste breathe, and imagine something new» («Respirez, simplement, respirez, et imaginez quelque chose de nouveau »).

Le Déluge, qui efface la terre, mais aussi une première histoire humaine partie dans la mauvaise direction, nous ramène alors aux premiers moments de la Genèse, au moment où la terre se situait entre les eaux d'en-haut et les eaux d'en-bas ${ }^{21}$. Ces variations sur le Déluge et la Création du monde nous renvoient alors à l'idée de Commencement.

\section{Des figures de démiurge}

Ces « dramaturges de la peinture $»^{22}$ ont en effet en commun de se penser comme des créateurs. Romeo Castellucci est peut-être celui qui articule le mieux ce rapport entre recours à la matière brute et possibilité d'une création nouvelle à partir de cet informe, qui contient en germes tous les mondes possibles. Il écrit ainsi à propos de son travail :

Lorsque j'élabore un spectacle, un travail, je pars d'un petit carnet de notes que je recueille chaque jour. C'est un exercice quotidien. Je note sur ces pages toutes les sensations que la journée m'offre. Ce carnet plein de notes, de sensations et d'idées, constitue la matière fondamentale du travail qui va suivre. En le feuilletant, on s'aperçoit tout de suite que c'est un chaos, que c'est un vrai carnet de déchets (...). Donc chaque fois je me retrouve dans la même situation génératrice que Dieu; élaborer ce chaos pour pouvoir le faire sortir, pour pouvoir réunir ces possibilités. Au début chaque artiste sait que le plateau vide est la mer ouverte des possibilités et c'est en cela que consiste aussi la terreur de la scène. Ce n'est pas tant - en ce qui me concerne - la terreur du vide, mais la terreur du plein : il y a trop de choses, la quantité nous submerge. La matière est obscure ${ }^{23}$.

Castellucci décrit ainsi ses notes, sensations et idées à la fois comme "la matière fondamentale » de son travail, et « un chaos ». La matière de la peinture, quand elle se déverse sur scène - dans des spectacles aussi différents que Moses und Aron, la Tragedia Endogonidia ou Sur le concept du visage du fils de Dieu - semble alors venir faire écho à ce chaos originel, qu'il va falloir élaborer. L'obscurité de la matière doit ici être opposée à la clarté de l'expression, ou des idées; le metteur en scène retrouve cette obscurité dans l'opacité de la peinture-matière et sa capacité à ne pas faire immédiatement sens (Guillaume Cassegrain parle ainsi de "l'insensé d'un bégaiement engendré par la persistance de la matière opaque de la peinture $\left.{ }^{24} »\right)$.

La peinture-matière qui envahit la scène serait alors cette "mer ouverte des possibilités » à partir de laquelle il faut créer, et créer en Dieu. Créer en Dieu, c'est, à partir de cette matière informe, élaborer des divisions ou des séparations. Dieu en effet agit en peintre, qui sépare d'un trait la terre et le ciel, la nuit et le jour, la terre et la mer, avant de prendre du recul et décréter que cela est bien ${ }^{25}$. Mais, à la différence de Dieu, il semble important pour nos trois metteurs en scène de toujours garder en vue, ou du moins de toujours revenir à «la mer ouverte des possibilités ». Mondrian disait à 
propos de sa peinture : «Lorsque l'on représente quelque chose de percevable pour les sens on exprime quelque chose d'humain. Lorsque l'on ne représente pas les choses, il reste de la place pour le divin ${ }^{26}$. " Faire cohabiter sur le plateau matière informe et figures permettrait ainsi, finalement, de faire cohabiter l'humain et le divin. Or, qu'il s'agisse de Jan Fabre qui se représente en nouveau Christ, brandissant une croix sur les toits de Florence ${ }^{27}$, ou de Vincent Macaigne, qui orchestre tour à tour, dans ses deux derniers spectacles, Déluge et Annonciation, tous ces artistes qui font couler la peinture sur scène semblent bien souffrir de ce complexe du créateur-démiurge. L'arrivée de la peinture à grands flots, qui amène avec elle l'informe, contient en elle la possibilité d'une recréation. Sur scène, la fluidité de la matière rappelle celle, originelle, de la formation du monde.

\section{« Refaire monde » : la puissance d'images de la peinture-matière}

48 La place centrale qu'a peu à peu prise la peinture-matière dans des dramaturgies aussi différentes que celles de Vincent Macaigne, Romeo Castellucci ou Jan Fabre s'explique donc en partie par les infinies possibilités de signification que contient en elle cette matière encore non informée. Face à la rigidité du langage articulé - il s'agit pour la Societas de Romeo Castellucci de s'affranchir des formes déjà existantes, en les détruisant et en recréant à partir de leurs ruines ${ }^{28}$, pour Jan Fabre de sortir de la Renaissance et de ses cadres ${ }^{29}$, pour Vincent Macaigne de retrouver les forces vitales emprisonnées dans les grands textes ${ }^{30}$ - la peinture-matière permet de retrouver une " plasticité » mieux à même de suivre les capacités de transformations incessantes de la pensée. Sur leurs scènes, comme on l'a vu, la peinture peut être utilisée tour à tour comme icône, indice ou symbole. Lorsqu'elle est utilisée comme symbole, c'est souvent comme un symbole de résistance à l'ordre des choses : révolutions chez Macaigne, blasphème chez Castellucci, résistance à l'humanisme triomphant chez Jan Fabre. Les metteurs en scène utilisent alors, même quand ils l'utilisent pour faire sens, la capacité de résistance au sens de la peinture, qui, dans son essentielle liquidité, refuse le cadre ${ }^{31}$. Mais loin d'être uniquement un symbole de résistance à l'ordre et au cadre, la peinturematière est aussi mise en avant comme peinture par ces metteurs en scène, et plus exactement, comme matière dont ils exploitent toutes les caractéristiques (coulure, opacité, informe).

La recherche de ces caractéristiques permet d'expliquer en partie la frénésie de destruction que l'on observe dans certains spectacles - les metteurs en scène détruisant souvent les reproductions de tableaux qui peuplent leurs plateaux justement pour accéder à cette matière première. Macaigne érige cette frénésie de destruction en art poétique, au prétexte que toutes les histoires ont été dites, et que tout a déjà été créé. C'est peut-être dans son théâtre que la volonté de recréation après le chaos est la moins évidente, et le metteur en scène semble parfois s'adonner à un désespoir narcissique qui se complaît dans les ruines du présent. Cependant, certains signes donnent à penser que même lui ne s'en tient pas au chaos dont il se fait le champion. Sur la scène de Je suis un Pays, après que tout a été détruit et alors que le plateau est recouvert des débris de ce qui a eu lieu - peinture, eau, terre, etc. -, seules restes intactes les reproductions du Caravage suspendues sur les murs latéraux, alors que la petite fille incarnant la parole nouvelle proclame "l'avenir sera à nous". Il s'agit bien de reconstruire un monde nouveau, à partir du chaos retrouvé. 

au terme duquel les liquides doivent refaire surface. Il fait ainsi subir à ses performeurs des épreuves physiques d'une difficulté et d'une longueur presque insoutenables, pour retrouver le corps suintant qu'il appelle de ses vœux. Au terme de ses spectacles, c'est la scène elle-même qui finit par se rendre, jusqu'à suinter elle-aussi de matières - qu'il s'agisse d'eau, de faux sang ou de peinture - à partir de laquelle il sera possible d'« imaginer un nouveau monde ».

51 Romeo Castellucci, dans ses écrits théoriques, est peut-être enfin celui qui a le mieux théorisé ce rapport entre destruction et création, alors qu'il se définit lui-même comme un artiste iconoclaste, désireux de «mettre le feu aux archives de la tradition $»^{32}$. Ce désir iconoclaste est cependant d'un genre très particulier. La guerre iconoclaste de Romeo Castellucci est certes une guerre contre les formes existantes, qui empêchent de " dépasser la réalité " $^{33}$ en faisant surgir des idées nouvelles ; mais il ne s'agit en aucun cas d'arriver à un monde sans images. Il faut, à partir du chaos retrouvé qui nous redonne accès à la matière - et Castellucci lui-même rapproche cette matière du chaos originel ${ }^{34}$ - « refaire monde $»^{35}$. Il faut, en d'autres termes, libérer dans cette peinturematière sa puissance d'images.

problème est alors de remettre cette matière en forme. Fabre s'y attelle en plasticien, en recomposant à partir de figures de l'histoire de l'art, qu'il refond, déforme, recompose. Castellucci, lui, se fait scribe, et tente d'inventer à la fois les signes et la grammaire de cette nouvelle écriture, dans une combinatoire de hiéroglyphes qui rappelle $\operatorname{Artaud}^{36}$. Macaigne est quant à lui peut-être celui qui s'en tiendra le plus fermement à sa volonté première de mise à mort des images ; ce chaos retrouvé semble chez lui faire une place nouvelle au Verbe, quand on sait l'importance dans ses derniers spectacles de la figure de l'Annonciation.

La traversée de la matière brute n'entraîne donc pas un abandon de l'écriture, bien au contraire; que ce soit chez Macaigne où le Verbe semble sortir tout-puissant de la destruction du plateau, ou bien chez Fabre et Castellucci, qui reviennent à un avant du Verbe, et à la logique de l'image, il s'agit bien de trouver de nouvelles méthodes de composition théâtrales, et plus précisément d'arracher aux récits fondateurs auxquels tous s'attachent le fait théâtral, en dehors de toute narration, pour travailler ce que Planchon appelait déjà « l'espace mental » du théâtre.

\section{BIBLIOGRAPHIE}

ARTAUD, Antonin. Le théâtre et son double (1938), Paris, Gallimard, coll. Folio essais, 2014.

CASSEGRAIN, Guillaume. La coulure, histoire(s) de la peinture en mouvement, XIe-XXIe siècles, Paris, Editions Hazan, 2015.

CASTELLUCCI, Claudia et Romeo. Les Pèlerins de la matière, Théorie et praxis du théâtre - Ecrits de la Societas Raffaello Sanzio, Besançon, Les Solitaires intempestifs, 2001, 
FABRE Jan. L'histoire des larmes/L'Empereur de la perte/Le Roi du plagiat / Une tribu, voilà ce que je suis/ Je suis une erreur, Postfaces de Georges Banu, Sigrid Bousset et Luk Van de Dries, Paris, L'Arche, 2005.

MACAIGNE, Vincent. Je suis un Pays suivi de Voilà ce que jamais je ne te dirai, Paris, Actes Sud, 2018, Kobo, Adobe Digital Editions.

MICHEL, Régis. Posséder et détruire : stratégies sexuelles dans l'art d'Occident, Paris, Réunion des Musées nationaux, 2000.

MICHEL, Régis. La peinture comme crime ou « la part maudite » de la modernité, Paris, Réunion des Musées nationaux, 2001.

SCHNEIDER, Pierre. Petite histoire de l'infini en peinture, Paris, Hazan, 2001.

VEYNE, Paul. Mon musée imaginaire, Paris, Albin Michel, 2012.

\section{NOTES}

1. Roger Planchon, cité par Michel Bataillon in Un défi en province. Planchon: chronique d'une aventure théâtrale, 1972 - 1986, Paris, Marval, 2005, p 17.

2. Exposition présentée au musée du Louvre, Hall Napoléon, du 10 avril au 10 juillet 2000. Cette exposition a également donné lieu à un ouvrage écrit par le commissaire de l'exposition: Régis Michel, Posséder et détruire: stratégies sexuelles dans l'art d'Occident, Paris, Réunion des Musées nationaux, 2000.

3. Exposition présentée au musée du Louvre du 19 octobre 2001 au 14 janvier 2002. Cette exposition a également donné lieu à un ouvrage écrit par le commissaire de l'exposition : Régis Michel, La peinture comme crime ou «la part maudite " de la modernité, Paris, Réunion des Musées nationaux, 2001.

4. Guillaume Cassegrain, La coulure, histoire(s) de la peinture en mouvement, XIe-XXIe siècles, Paris, Editions Hazan, 2015, p. 204.

5. Vincent Macaigne, Je suis un Pays suivi de Voilà ce que jamais je ne te dirai, Paris, Actes Sud, 2018, Kobo, Adobe Digital Editions (non paginée) : « Marie, une cagoule sur le visage, déboule en hurlant. Elle vide un seau d'excréments sur la journaliste. »

6. Ibid.

7. Karl Marx, Le 18 Brumaire de Louis Bonaparte (1851) : " Hegel fait quelque part cette remarque que tous les grands événements et personnages historiques se répètent pour ainsi dire deux fois. Il a oublié d'ajouter : la première fois comme tragédie, la seconde fois comme farce.» (éd. numérique, collection «Les classiques des sciences sociales", développée par la Bibliothèque Paul-Émile-Boulet de l'Université du Québec à Chicoutimi, p 13). Document consultable en ligne : <http://classiques.uqac.ca/classiques/Marx_karl/18_brumaine_louis_bonaparte/

18_brumaine_louis_bonaparte.pdf>

8. Ibid.

9. Guillaume Cassegrain, La coulure, histoire(s) de la peinture en mouvement, XIe-XXIe siècles, Paris, Editions Hazan, 2015, p. 102.

10. Hans-Thies Lehmann, «Ob-scaena, The tragic » in Mount Olympus - To glorify the cult of tragedy, program (je traduis).

11. «Je pense que la foi, comme on peut la voir dans la grande histoire de la mystique, c'est l'exercice du doute entendu comme discipline. " (Entretien de Romeo Castellucci avec Augustin Trappenard dans l'émission Boomerang, « Romeo Castellucci Dieu merci », France Inter, 10 janvier 2019. URL : <https://www.franceinter.fr/emissions/boomerang/boomerang-10-janvier-2019> 
12. On rappelle que pour Charles Sanders Peirce, « un Indice est un signe qui fait référence à l'objet qu'il dénote en vertu du fait qu'il est réellement affecté par cet Objet », tandis qu' « un Symbole est un signe qui se réfère à l'objet qu'il dénote en vertu d'une loi » (Elements of Logic (1903) in Charles S. Peirce, Collected Papers. Vol. II, Cambridge : Harvard University Press, 1932).

13. On rappelle qu'un signe renvoie à son objet de façon iconique lorsqu'il ressemble à son objet.

14. Guillaume Cassegrain, La coulure, histoire(s) de la peinture en mouvement, XIe-XXIe siècles, ibid, p.

28.

15. Claudia et Romeo Castellucci, Les Pèlerins de la matière, Besançon, Les Solitaires intempestifs, 2001, pp. 34-35: «L'acteur n'est pas celui qui fait, mais celui qui reçoit. C'est celui qui est " enlevé », celui dont le corps est consumé par le regard ardent des spectateurs et dont le sang auriculaire coule à flots, en hémorragie, libation au plateau. »

16. Le geste révolutionnaire étant symbolisé, dans ce spectacle comme dans Je suis un Pays, par la projection et le déversement de peinture noire. Liza et Clara, les deux révolutionnaires de la pièce, ont ainsi mis à mort Sofia Burini en l'aspergeant de peinture noire, cette peinture ayant également servi à détruire les reproductions des tableaux du Caravage exposées au mur.

17. Antonin Artaud, Le Théâtre et son double (1938), Paris, Gallimard, Folio essais, 2014, p. 15.

18. On pourrait aussi faire de nombreux parallèles entre les personnages du roman et les personnages de Macaigne. Sofia Burini, richissime collectionneuse qui avait rêvé d'apporter la lumière au plus grand nombre, semble ainsi inspirée de Settembrini (Macaigne pousse la parodie jusque dans les noms), figure d'un humanisme éclairé, fervent défenseur des Lumières et des droits de l'homme, lui-même comparé à la figure de Prométhée, qui apporta le feu et la connaissance aux hommes. Liza, la fille de Sofia Burini, partage de nombreux traits avec Hans Castorp; comme lui, elle est en quête d'un monde meilleur, remplie d'idéaux humanistes, et comme lui (décrit comme un être assez médiocre et indécis), elle est incapable d'aller au bout de sa révolution. La pièce s'arrête alors qu'elle reconnaît son échec et qu'elle s'apprête à mourir, tout comme le roman s'arrête alors que Castorp est envoyé sur le front allemand de la première guerre mondiale - Macaigne qualifiant lui-même son spectacle de spectacle « d'avant-guerre ».

19. Extrait de la présentation du spectacle par Eric Vautrin, dramaturge du Théâtre VidyLausanne : <http://www.altermachine.fr/en-manque>

20. Je suis sang a pour sous-titre "Conte de fées médiéval », et les personnages principaux de L'histoire des larmes, le chevalier du désespoir et la Dame au Rocher, respectivement en armure et en coiffe, sont tous deux issus de l'imaginaire médiéval flamand.

21. La Bible, Traduction officielle liturgique, Paris, Mame, 2018, p. 11: «Dieu dit: «Qu'il y ait une étendue entre les eaux pour les séparer les unes des autres!» Dieu fit l'étendue et sépara ainsi l'eau qui est au-dessous de l'étendue de celle qui est au-dessus. Cela se passa ainsi. »

22. Nous appelons «dramaturges de la peinture " ces metteurs en scène et dramaturges qui prennent la peinture comme modèle de création, c'est-à-dire qu'ils pensent les différents éléments de leurs écritures scéniques - cadre, figures, organisation spatiale, et surtout matière comme ils penseraient les éléments d'une peinture.

23. Claudia et Romeo Castellucci, Les Pèlerins de la matière, op. cit., pp. 114-115.

24. Guillaume Cassegrain, La coulure, histoire(s) de la peinture en mouvement, XIe-XXIe siècles, op. cit., p. 10.

25. La Bible, Traduction officielle liturgique, op. cit., p. 11 : « Dieu vit que c'était bon. »

26. Mondrian, cité par Pierre Schneider, Petite histoire de l'infini en peinture, Paris, Hazan, 2001, p. 183.

27. Voir l'exposition Jan Fabre. Spiritual Guards qui a eu lieu à Florence en 2016.

28. Claudia et Romeo Castellucci, Les Pèlerins de la Matière, op. cit., p. 31 : « Le caractère essentiel de l'iconoclastie, en effet, est de transformer quelque chose qui avant avait une forme et qui maintenant en prend une autre.» 
29. Jan Fabre, L'histoire des larmes/L'Empereur de la perte/ Le Roi du plagiat / Une tribu, voilà ce que je suis/ Je suis une erreur, Postfaces de Georges Banu, Sigrid Bousset et Luk Van de Dries, Paris, L'Arche, 2005, p. 47 : «Le corps suintant, fondant, succulent du chevalier du désespoir est un anachronisme aujourd'hui. (...) Nous vivons de nouveau aux temps de la Renaissance, sous l'œil omniscient de la science qui veut tout déchiffrer et analyser. "

30. «1964)rmanceis. $\mathrm{n}$ des Musées nationaux, 2001. $\mathrm{t}$ de sa double nature, il ajoute pourtant un prestige singulier. hiéroglyphes, où Je ne dispose pas du corpus de textes au moment où je commence à travailler avec les acteurs. Je me lance dans un seul texte dont la problématique m'intéresse (...). En fait, j'aime être libre et penser que, lorsque je commence à travailler sur un projet, je peux tout me permettre. " (Entretien avec Vincent Macaigne, festival d'Avignon 2011, téléchargeable depuis la page de présentation du spectacle : <https://www.festival-avignon.com/ $\mathrm{fr} /$ spectacles/2011/au-moins-j-aurai-laisse-un-beau-cadavre>

31. Cet écoulement de peinture semble d'ailleurs aussi être une manière de résister à l'hypertechnicité et l'hypertechnologie qui guettent les scènes. Chez les trois metteurs en scène ici étudiés, ce combat a souvent lieu au sein même de leurs plateaux. On le voit en particulier chez Castellucci qui mêle recours à des machines et à des machineries extrêmement sophistiquées (on pense au serpent articulé de Moses und Aaron), élaboration d'une dramaturgie qui doit être précise parfois à la seconde près, et déversement immaîtrisé de peinture brute.

32. Claudia et Romeo Castellucci, Les Pèlerins de la matière, op. cit., p. 100.

33. Claudia et Romeo Castellucci, Les Pèlerins de la Matière, op. cit., p. 23.

34. Claudia et Romeo Castellucci, Les Pèlerins de la Matière, op. cit., pp. 114-115: « Chaque fois je me retrouve dans la même situation génératrice que Dieu; élaborer ce chaos pour pouvoir le faire sortir, pour pouvoir réunir ses possibilités. "

35. Claudia et Romeo Castellucci, Les Pèlerins de la Matière, op. cit., p. 23.

36. Antonin Artaud, Le théâtre et son double, Paris, Gallimard (folio essais), 2014, p. 59 : « Et une autre idée abstraite ou attitude d'esprit pourrait être représentée par quelques-uns des innombrables symboles de l'Ecriture, exemple : le trou d'aiguille à travers lequel le chameau est incapable de passer. On voit que ces signes constituent de véritables hiéroglyphes, où l'homme, dans la mesure où il contribue à les former, n'est qu'une forme comme une autre, à laquelle, du fait de sa double nature, il ajoute pourtant un prestige singulier. »

\section{RÉSUMÉS}

Le tournant des années 2000 marque l'arrivée à grands flots de la peinture sur scène, en une reprise - qui est aussi une réécriture - des pratiques de performance des années 1970. Il s'agira de voir comment des metteurs en scène aussi différents que Romeo Castellucci, Jan Fabre ou Vincent Macaigne, exploitent les caractéristiques matérielles de la peinture (opacité, coulure, informe) qui, sur leurs plateaux, s'impose comme une forme de résistance au cadre et au langage articulé. Simultanément, nous verrons comment ces metteurs en scène-plasticiens se saisissent des potentiels de la peinture liquide, et notamment sa capacité à faire déluge, pour rejouer l'épisode de la création du monde et renouer avec ce que l'on s'imagine être le chaos originel. 
INDEX

Mots-clés : Peinture, scènes contemporaines, Romeo Castellucci, Jan Fabre, Vincent Macaigne, révolte, déluge, écritures scéniques 